\title{
4 Auswirkungen der digitalen Transformation auf den Wettbewerb
}

\author{
M. Böhm, S. Müller, H. Krcmar, I. Welpe
}

Die Bedeutung einer technologischen Innovation für eine Branche ist schwer im Voraus abzuschätzen. Diese Schwierigkeit kann leicht dazu führen, dass sich Entscheider in Unternehmen erst dann mit den Implikationen einer neuen, digitalen Technologie für das eigene Unternehmen und die Branche auseinandersetzen, wenn deren Auswirkungen bereits spürbar sind. Anhand der Ergebnisse einer Umfrage unter IT-Verantwortlichen aus den USA und Deutschland sowie von Praxisbeispielen wird gezeigt, welche Veränderungen sich auf Branchen- und Unternehmensebene durch die Digitalisierung vollziehen und wie diese Veränderungen von Entscheidern bewertet werden. ${ }^{2}$

\footnotetext{
${ }^{2}$ Die hier vorgestellten Ergebnisse beruhen auf einer Studie (Müller, Böhm, Schröer, Bakhirev, Baiasu, Krcmar \& Welpe, 2016) im Auftrag der Expertenkommission Forschung und Innovation.
}

(C) Der/die Autor(en) 2018

G. Oswald und H. Krcmar (Hrsg.), Digitale Transformation, Informationsmanagement und digitale Transformation, https://doi.org/10.1007/978-3-658-22624-4_4 


\subsection{Einleitung}

Die Auswirkungen der Digitalisierung auf die Wettbewerbsbedingungen, die Wettbewerbsstruktur und die angebotenen Produkte und Dienstleistungen innerhalb einer Branche sind nicht immer offensichtlich. Die Transformationsprozesse innerhalb derer Unternehmen versuchen, die Potentiale des Einsatzes innovativer, digitaler Technologien für sich nutzbar zu machen, können Wettbewerbern lange verborgen bleiben und ihre Bedeutung für den Wettbewerb schwer abzuschätzen sein. Die Bedeutung einer Innovation in der Digitaltechnik oder des Einsatzes von Digitaltechnik für eine Branche ist schwer im Voraus abzuschätzen. Diese Schwierigkeit kann leicht dazu führen, dass sich Entscheider in Unternehmen erst dann mit den Implikationen einer neuen, digitalen Technologie für das eigene Unternehmen und die Branche auseinandersetzen, wenn deren Auswirkungen bereits spürbar sind. Leicht laufen Unternehmen Gefahr, auf Veränderungen in der eigenen Branche reagieren zu müssen, anstatt sie zu gestalten.

Anhand der Ergebnisse einer Umfrage unter 380 Unternehmensvertretern in Deutschland und den USA sowie einer Reihe von Praxisbeispielen illustriert dieser Beitrag, mit welchen Veränderungen Unternehmen und ganze Branchen zukünftig durch die Digitalisierung konfrontiert sein werden.

\subsection{Digitale Transformation auf Unternehmens- und Branchenebene}

Die Umfrage wurde zwischen dem 14. und 22. Januar 2016 unter deutschen und USamerikanischen Unternehmen durchgeführt. Pro Land haben jeweils 190 Unternehmen an der Befragung teilgenommen. Die Zielgruppe waren Führungskräfte, die an unternehmensstrategischen Entscheidungen beteiligt sind, bzw. Einblick in diese haben. Im Idealfall handelte es sich dabei um Geschäftsführer, Vorstände, Aufsichtsräte oder Personen auf C-Level. Zulässig waren auch Bereichs- und Abteilungsleiter sowie Führungskräfte mit ITVerantwortung. Ein wesentliches Kriterium war, dass sich die Teilnehmer bereits mit dem Thema Digitalisierung beschäftigt haben, um den Fragebogen sinnvoll ausfüllen zu können. Hierfür wurde dem Fragebogen eine Selektionsfrage hinzugefügt. Auf Basis der Selektionsfrage wurden 460 Teilnehmer der US-Umfrage und 162 Teilnehmer der Umfrage in Deutschland von der Analyse ausgeschlossen. Von den eingeladenen Personen haben 115 die Umfrage vorzeitig abgebrochen.

Die Umfrageergebnisse vermitteln den Eindruck, dass Unternehmen den zunehmenden Einfluss digitaler Technologien wahrnehmen. Wie Abbildung 1 zeigt, stimmt die Mehrheit der 
Umfrageteilnehmer der Hypothese zu, dass digitale Technologien große Auswirkungen auf die Wirtschaft haben werden. Ein Großteil der befragten Unternehmen prognostiziert große Auswirkungen auf die eigene Branche sowie das eigene Unternehmen, unabhängig davon, ob das Unternehmen bereits eigene Digitalisierungsaktivitäten durchführt. Hierbei unterscheiden sich die Einschätzungen zwischen deutschen und US-amerikanischen Umfrageteilnehmern nur geringfügig. Dies zeigt, dass dem überwiegenden Teil der Unternehmen aus den beiden Stichproben die Tragweite der Entwicklungen im Bereich der digitalen Technologien bewusst ist.

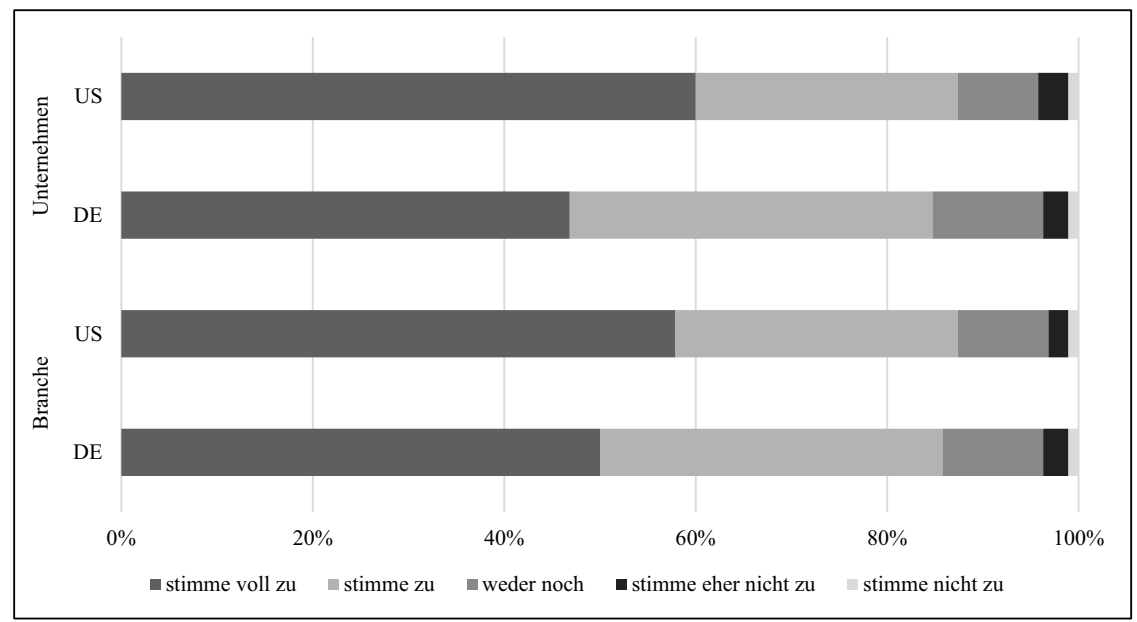

Abbildung 1: Wahrgenommener Einfluss digitaler Technologien (Quelle: Eigene Darstellung $\left(\mathrm{n}_{\mathrm{DE}}=190, \mathrm{n}_{\mathrm{US}}=\right.$ 190))

\subsubsection{Digitale Transformation auf Unternehmensebene}

Beispiele dafür, wie Unternehmen digitale Technologien nutzen können, um sich gegenüber ihren Wettbewerbern zu differenzieren, gibt es in praktisch jeder Branche. So zeigt der Einzelhandel, wie sich durch den innovativen Einsatz digitaler Technologien verschiedene Kanäle für die Kundeninteraktion nahtlos verknüpfen lassen, um dem Kunden ein interaktives Einkaufserlebnis zu bieten (weitere Beispiele digitaler Transformation auf Unternehmensebene werden in den Kapiteln 9 und 10 vorgestellt).

Ein illustratives Beispiel ist Burberry, ein weltweit bekanntes, britisches Modelabel, das 1856 gegründet wurde. Popularität erlangte das Label in den 20er und 30er Jahren durch seine karierten Trenchcoats. Die wachsende Marktmacht von Fast-Fashion-Händlern wie Zara, Topshop oder H\&M setzte das Unternehmen zunehmend unter wirtschaftlichen Druck. Die Kol- 
lektionen in den Fast-Fashion-Läden änderten sich teilweise im 3-Wochen-Rhythmus und boten ähnliche Kleidungsstücke für ein Zehntel des Preises an (Hagan, 2015). Im Jahr 2006 blieb das Unternehmen signifikant hinter den jährlichen Wachstumszahlen der Konkurrenz zurück. Während das durchschnittliche jährliche Wachstum in der Branche 12-13 \% betrug, wuchs Burberry jährlich nur um 1-2 \% und konnte weder in der Wachstumsrate, noch bei den Innovationen im Produkt- und Serviceangebot mithalten (Capgemini Consulting, 2012a).

Um die Wettbewerbsfähigkeit des Unternehmens zu steigern, wurde ein umfangreiches Transformationsprogramm gestartet, welches sich stark auf digitale Technologien fokussierte. Neben einem umfassenden, internen digitalen Transformationsprozess, bei dem ein unternehmensweites SAP-System ausgerollt wurde, um interne Geschäftsprozesse in einem Informationssystem zu integrieren (Capgemini Consulting, 2012b), wurde eine digitale Transformation des Markenauftritts angestoßen. So wurden 60 \% des Marketingbudgets in digitale Kanäle investiert, während der Branchendurchschnitt bei 15-20 \% lag (Barrett \& Bradshaw, 2011). Nach der Umsetzung der beschriebenen Maßnahmen entwickelte sich Burberry zu einem hochprofitablen Unternehmen mit einer Umsatzrendite von 14,1 \% (Burberry, 2017).

Mit der digitalen Transformation ging eine Neufokussierung auf die Zielgruppe der sogenannten Millennials einher, digital affinen Kunden zwischen 20 und 30 Jahren (Capgemini Consulting, 2012b). Die Digitalisierung des Marketings beinhaltete eine stärkere Ausrichtung auf die Präsentation von Produkten über soziale Medien wie Facebook und Twitter. Modenschauen werden live über YouTube und Facebook übertragen und von einem renommierten Fotografen auf dem Burberry-eigenen Kanal der Fotoplattform Instagram festgehalten. TwitterNutzer können neue Kollektionen ,backstage“ sehen, bevor sie auf dem Laufsteg präsentiert werden (Dishman, 2011). Burberrys Ziel war es, sich von einer Marke zu einer sozialen Plattform zu wandeln. Dazu gehört, dass Fans und Followern der Marke die Möglichkeit gegeben wird, über die eigens entwickelte Burberry-Plattform Bilder mit Burberry-Produkten hochzuladen oder - analog zu mi adidas oder NIKEiD - Produkte aus den aktuellen Kollektionen individuell zu gestalten und anschließend zu bestellen (Hagan, 2015).

Burberrys Vision ist die von einem Omni-Channel-Retailer, der seinen Kunden integrierte Produkt- und Serviceleistungen anbietet. Hierzu mussten die Online-Kanäle mit den OfflineKanälen verknüpft werden. Das Verkaufspersonal in den Ladengeschäften wurde mit iPads ausgestattet, um Kunden die Möglichkeit zu bieten, auf den gesamten Online-Store zuzugreifen und eventuell nicht vorhandene Produkte bequem zu sich nach Hause zu bestellen. Eine weitere Maßnahme war die Einführung des sogenannten „Retail Theatre“, durch das die Kunden in den 
Läden Modenschauen per Live-Stream weltweit verfolgen und direkt nach der jeweiligen Modenschau die präsentierten Produkte per iPad bestellen können (Capgemini Consulting, 2012b).

\subsubsection{Digitale Transformation auf Branchenebene}

Das Thema Omni-Channel-Vertrieb hält auch im Lebensmittelhandel Einzug. Das Ziel ist es dem Kunden den Alltag zu erleichtern indem ihm Routineeinkäufe abgenommen werden. Insbesondere in Großstädten entstehen Lieferdienste für Lebensmittel. Auch die großen Handelskonzerne bieten vermehrt Online-Bestellmöglichkeiten an. Als große Herausforderung ist hierbei allerdings die Zustellung auf Grund der heterogenen Produktanforderungen insbesondere hinsichtlich der Temperaturen während des Transports und der Lagerung zu sehen. Die Handelskonzerne setzen dabei auf Omni-Channel Strategien, die auch eine Abholung der Waren in den Filialen erlauben. Zu diesem Zweck ergänzen erste Lebensmittelhändler ihr Filialgeschäft um einen Online-Shop als digitalen Vertriebskanal. Der Online-Kanal ist transparent in das stationäre Geschäft eingebunden, sodass Kunden die Produkte online (bspw. über ein Smartphone) bestellen können und sich diese entweder liefern lassen oder in ausgewählten Filialen mit Fulfillment-Centern fertig gepackt abholen können.

Ein weiteres Beispiel anhand dessen sich die durch Digitalisierung hervorgerufenen Veränderungen auf der Branchenebene illustrieren lassen, ist die Medienbranche. Seit Jahren geht der Umsatz mit Druckerzeugnissen wie Zeitschriften, Büchern und Katalogen aber auch Postkarten, Flugblättern und Plakaten weiter zurück. Diese Produkte werden zunehmend als digitale Medien angeboten. Infolgedessen schreitet die Konsolidierung der Druckereien weiter voran während gleichzeitig der Umsatz von Onlinedruckereien steigt. Der Trend in der Druckindustrie geht hin zu kleineren Losgrößen und dem Individualdruck, bei dem Endverbraucher ihr individuelles Druckprodukt gestalten und per Post geliefert bekommen (BVDM, 2015). In diesem Bereich haben sich verschiedene Nischenanbieter etabliert, die beispielsweise den Posterdruck von digitalen Bildern anbieten.

Auch die klassischen Verlage stehen vor der Herausforderung, sich in der digitalen Welt zu etablieren. Mit dem Internet und interaktiven Online-Diensten wie Facebook oder Twitter hat sich das Konsumverhalten für Nachrichten verändert. So geht der Absatz von Zeitungen in Deutschland kontinuierlich zurück (seit 2003 um ca. $23 \%$ (Statista, 2015)). Um dieser Entwicklung zu begegnen, setzen die Verlage auf Onlineausgaben ihrer Zeitungen und Zeitschriften, kostenpflichtige oder durch gezielte Werbung finanzierte Nachrichten auf ihren OnlinePlattformen, sowie die Schaffung einer Marke, welche für Qualitätsnachrichten steht 
(Burgelman, Siegel, \& Luther, 2014). Der Trend in der Nachrichtenbranche geht weiter zu individuellen Nachrichten. Auf Basis des eigenen Nutzerprofils stellen Aggregationsdienste wie beispielsweise Apple News ein individuelles Portfolio an Nachrichten zusammen. Auch Facebook will durch intelligente Algorithmen individuelle Nachrichten in sein Angebot integrieren und so zur führenden Nachrichtenplattform werden (Kim, 2014).

Veränderungen zeigen sich auch in der Musik- und Filmbranche. So gewinnen StreamingDienste wie Spotify für Musikangebote oder Netflix für Filme und Serien immer mehr an Zuspruch. Getrieben wird diese Entwicklung durch die Verfügbarkeit von Breitbandinternet sowie dem veränderten Nutzerverhalten. Streaming-Dienste bieten Zugang zu einem umfangreichen Programmangebot, losgelöst von festen Sendezeiten. Kunden können sich somit ihr individuelles Programm gestalten. Den Trend zu individuellen Inhalten adressieren auch verschiedene Nischenanbieter, für die digitale Technologien die Grundlage bilden, um kosteneffizient Spartenprogramme für besondere Interessensgruppen zu produzieren. So haben sich insbesondere auf die Videoberichterstattung im Sportbereich spezialisierte Firmen wie clipmyhorse.tv (Reitsport) oder fupa.tv (Amateurfußball) etabliert (weitere Beispiele digitaler Transformation auf Branchenebene werden in den Kapiteln 1 und 12 vorgestellt).

Während die Vernetzung es ermöglicht, bisher ungenutzte Wertschöpfungsbeiträge zu heben, bilden sich in vielen Branchen digitale Plattformen heraus, die eine ganz eigene Wettbewerbsdynamik besitzen. Netzwerkeffekte ermöglichen Plattformbetreibern, besonders starke und dominante Wettbewerbspositionen einzunehmen. Durch theoretische Modelle lässt sich zeigen, dass unter bestimmten Voraussetzungen die Dominanz so ausgeprägt ist, dass Plattformbetreiber ihre Marktposition selbst dann noch behaupten können, wenn neue Akteure mit qualitativ besseren Produkten oder Dienstleistungen versuchen, in den Markt einzudringen (Zhu \& Iansiti, 2012). In der Praxis besteht dadurch die Möglichkeit, dass Unternehmen ihre beherrschende Stellung ausnutzen, um eigene Angebote zu bevorzugen. Dieser Vorwurf wurde bspw. von Seiten der Europäischen Kommission gegenüber Google untersucht. Im Bereich der Einkaufsportale wurde Google vorgeworfen, das eigene Preisvergleichsportal in den Suchergebnissen systematisch bevorzugt zu platzieren und an prominenter Stelle anzuzeigen. Darüber hinaus wurde dem Unternehmen vorgeworfen, die Kriterien, die Google bei anderen Betreibern für die Abwertung im Ranking der Ergebnisse nutzt, nicht auf Google Shopping anzuwenden. Als Beispiel für die Auswirkungen führt die Europäische Kommission an, dass bei Googles erstem Shoppingportal Froogle (im Unterschied zum aktuellen Angebot Google Shopping) die Bevorzugung nicht zum Einsatz kam und das Angebot deshalb von Verbrauchern weniger 
nachgefragt wurde. Dagegen führt die aktuelle Bevorzugung von Google Shopping zu höheren Wachstumsraten. Als Folge sieht die Europäische Kommission negative Auswirkungen für Kunden und Innovatoren, da die Bevorzugung von Google Shopping Konkurrenten von Innovationsaktivitäten in diesem Bereich abhält (Europäische Kommission, 2015). Inzwischen wurde gegen Google eine Strafzahlung in Höhe von 2,42 Mrd. € verhängt (manager magazin, 2017). Der Fall zeigt, welche Risiken für Wettbewerber ohne selbstbetriebene Plattform bei der Teilnahme an plattformbasierten Märkten bestehen. Insbesondere bei datengetriebenen Diensten besteht die Gefahr der Ausnutzung von Schlüsselpositionen (OECD, 2015).

\subsection{Auswirkungen digitaler Transformation}

Im Folgenden sollen nun konkrete mögliche Auswirkungen näher erläutert werden. Von den Umfrageteilnehmern werden dazu insbesondere Veränderungen in den Wertschöpfungsnetzwerken durch neue Wettbewerber sowie zunehmende Abhängigkeiten gezählt (vgl. Abbildung 2). So gehen $73 \%$ der Unternehmen davon aus, dass neue Wettbewerber auf den Markt treten werden, was einen stärkeren Konkurrenzkampf mit sich ziehen wird. Dies wird auch zu einer Veränderung in den Wertflüssen zwischen den verschiedenen Akteuren führen.

Die Einschätzung der Umfrageteilnehmer deckt sich mit der Beobachtung, dass durch technologische Innovationen und neue Betriebskonzepte für IT-Infrastrukturen und Applikationslandschaften Eintrittsbarrieren in vielen Märkten gesenkt werden. So dienen das Cloud-Computing und insbesondere Software-as-a-Service (SaaS) als „Enabler“ für Startups. Ein junges Unternehmen kann bspw. auf hoch skalierbare Rechen-, Speicher-, oder Netzwerkressourcen nach Bedarf zugreifen, ohne umfangreiche Investitionen in und den Aufbau von Kompetenzen für den Betrieb leistungsfähiger IT-Infrastrukturen leisten zu müssen. Dies ermöglicht es, innovative Geschäftsideen früher am Markt zu erproben und gleichzeitig das Investitionsrisiko zu reduzieren. Ein Beispiel für ein solches Startup ist das amerikanische Unternehmen Alpha ${ }^{3}$. Alpha vergleicht und vermittelt über eine Webseite Finanzierungsoptionen verschiedener Anbieter für das Leasing von Maschinen, Haushaltsgeräten und Unterhaltungselektronik. Dabei vertraut das Unternehmen fast ausschließlich auf Cloud-basierte Dienste, angefangen bei den Google Apps als Bürosoftwarepaket und E-Mail-System, über Dropbox für die interne und externe Bereitstellung von Dateien bis hin zu Salesforce CRM für das Kundenbeziehungsmanagement. Die Verwendung von Cloud-Diensten senkte die finanziellen Einstiegsbarrieren erheblich, da keine Investitionen für Infrastruktur notwendig waren. Die Servicequalität der Cloud-

\footnotetext{
${ }^{3}$ Der Name des Unternehmens wurde anonymisiert.
} 
Dienste erreicht ein höheres Niveau, beispielsweise hinsichtlich der Verfügbarkeit und Datensicherheit, als dies das Startup selbst im Eigenbetrieb erbringen könnte. Durch die Nutzung von Cloud-Computing konnte das Unternehmen zudem einen erheblich größeren Anteil der zur Verfügung stehenden finanziellen und personellen Ressourcen für die Realisierung der Geschäftsidee einsetzen. Laut Aussage des Gründers hätte Alpha ohne Cloud-Computing nicht so schnell gegründet werden und wachsen können.

Ein Drittel der Umfrageteilnehmer geht außerdem davon aus, dass ihr Unternehmen in den kommenden fünf Jahren in zunehmendem Maße von anderen Unternehmen mit einer zentralen Rolle im Wertschöpfungsnetzwerk, wie beispielsweise Plattform- oder Marktplatzanbietern, abhängig sein werden. Dabei ist auffällig, dass die Sorge vor Verdrängung und der Bildung von Monopolen in den USA deutlicher ausgeprägt ist als in Deutschland. Während $68 \%$ der amerikanischen Umfrageteilnehmer mit der Entstehung von Monopolen rechnen, glauben dies nur $53 \%$ der deutschen Befragten. Diese Einschätzungen verdeutlichen die Notwendigkeit, die Entwicklung monopolähnlicher Strukturen zu beobachten und deren Auswirkungen auf den Wettbewerb zu analysieren. Darüber hinaus müssen auch staatliche Institutionen diese Entwicklungen verfolgen und neue Phänomene wie Monopolstellungen in Plattformökonomien untersuchen und gegebenenfalls regulatorisch eingreifen.

Anhand des Onlinehändlers Amazon lässt sich beobachten, wie schnell eine ganze Branche abhängig von einer einzelnen Plattform werden kann. In den USA ist der Anteil Amazons am Umsatz des gesamten Onlineeinzelhandels von 25 \% im Jahr 2012 auf 43 \% im Jahr 2016 gestiegen ${ }^{4}$. Das enorme Wachstum ist unter anderem auf die steigende Zahl an Einzelhändlern zurückzuführen, die ihre Produkte über die Amazon-Plattform verkaufen. Inzwischen bietet Amazon über 64.000 Händlern eine Plattform für den Verkauf ihrer Produkte ${ }^{5}$. Die den Händlern angebotenen Dienstleistungen umfassen neben der Zahlungsabwicklung, der Lagerhaltung und der Verpackung auch den Versand der Ware sowie die Rückabwicklung des Verkaufs im Falle einer Rücksendung der Ware durch den Kunden. Ähnlich wie bei der Buchung von Rechen- oder Speicherressourcen in Form von Cloud-Diensten, braucht ein Händler keine eigene Infrastruktur mehr aufzubauen, um Waren über das Internet zu verkaufen. Im Jahr 2015 wurden $44 \%$ der weltweit auf Amazon gelisteten Produkte von unabhängigen Händlern über den Amazon-Marketplace verkauft ${ }^{6}$. Im Jahr 2016 haben die auf dem Marketplace operierenden Händler

\footnotetext{
${ }^{4}$ www.businessinsider.de/amazon-accounts-for-43-of-us-online-retail-sales-2017-2, zugegriffen am 10.08.2017

${ }^{5}$ www.sistrix.de/news/anzahl-der-irrelevanten-haendler-auf-amazon-de-steigt/, zugegriffen am 10.08.2017

${ }^{6}$ www.theguardian.com/technology/2015/jun/23/amazon-marketplace-third-party-seller-faustian-pact, zugegriffen am 10.08.2017
} 
mehr als 2 Milliarden Artikel verschickt ${ }^{7}$. Durch die Reichweite die die Amazon-Plattform aufgrund ihrer dominierenden Stellung in vielen Märkten hat, wird es für Onlineeinzelhändler immer schwieriger, Amazon nicht in den eigenen Vertriebskanalmix einzubinden.

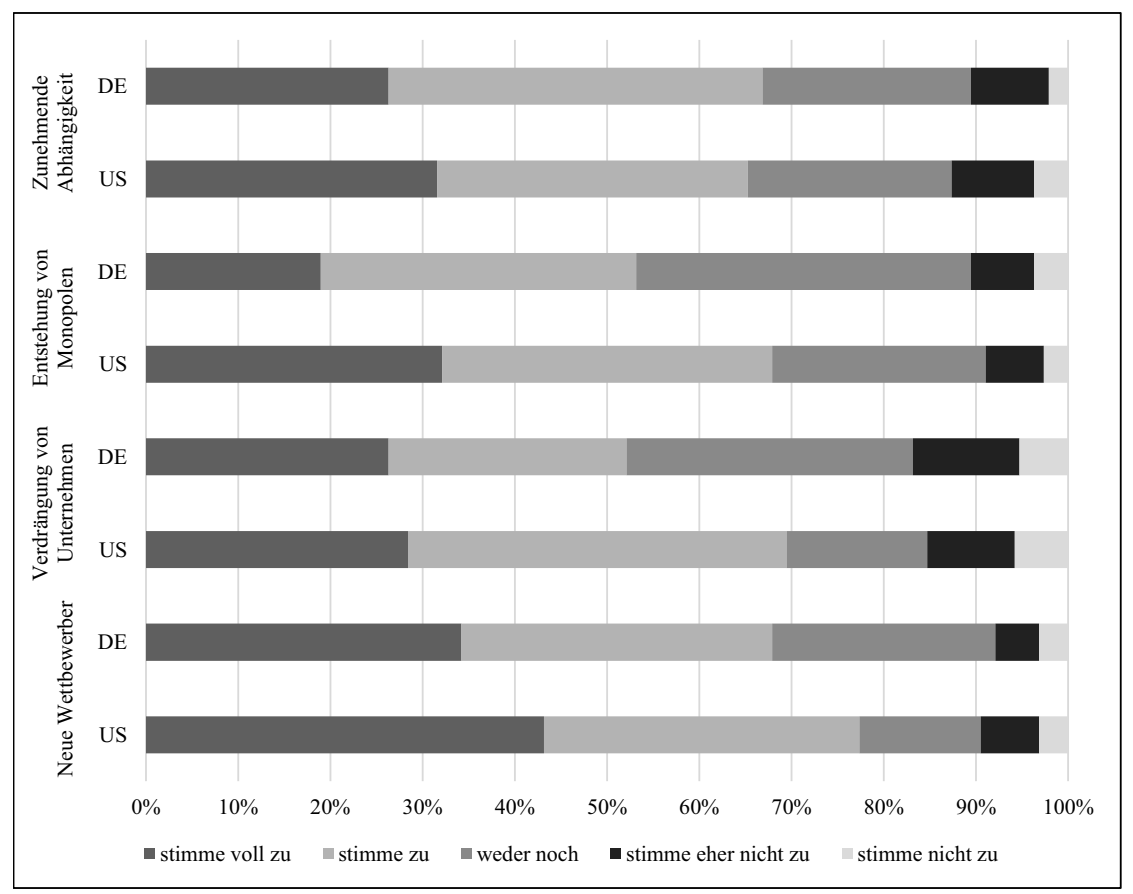

Abbildung 2: Auswirkung der digitalen Transformation (Quelle: Eigene Darstellung $\left(\mathrm{n}_{\mathrm{DE}}=190, \mathrm{n}_{\mathrm{US}}=190\right)$ )

Vor dem Hintergrund der erwarteten Veränderungen sieht ein Großteil der Unternehmen die Digitalisierung sowohl als große Herausforderung als auch als große Chance (vgl. Abbildung 3). Dabei ist auffällig, dass weniger amerikanische Unternehmen in der Digitalisierung eine Herausforderung sehen (48 \%) als deutsche Unternehmen (76 \%). Dieser Umstand kann möglicherweise auf kulturelle Unterschiede zurückzuführen sein. Des Weiteren fällt auf, dass die US-amerikanischen Unternehmen, die sich in der Planungs- oder Umsetzungsphase ihrer ersten Digitalisierungsaktivitäten befinden, die Herausforderungen als weniger gravierend einschätzen (44\%) als die US-amerikanischen Unternehmen, welche bereits erste Digitalisierungsaktivitäten realisiert haben (60\%). Dies könnte darauf zurückzuführen sein, dass Unter-

\footnotetext{
${ }^{7}$ www.amalyze.com/2017/01/12/marketplace-haendler-versand-durch-amazon-2016-2-milliarden-artikel-verschickt/, zugegriffen am 10.08.2017
} 
nehmen im Vorfeld die Herausforderungen, die sich mit der Einführung von Digitalisierungsinitiativen ergeben, unterschätzen und sich erst nach erfolgten ersten Projekten intensiv damit auseinandersetzen konnten.

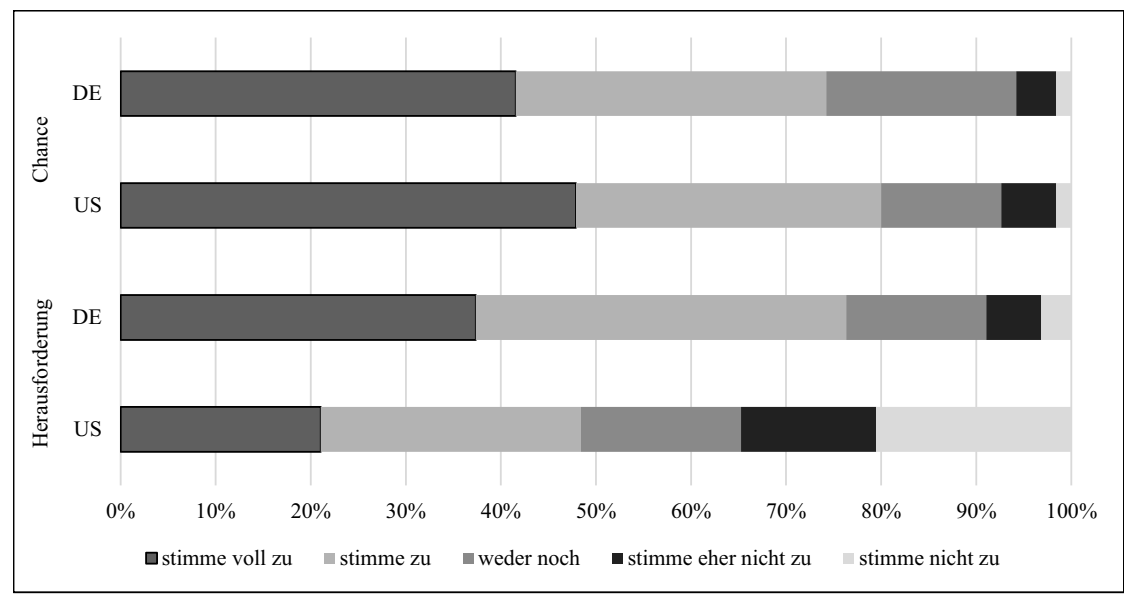

Abbildung 3: Digitalisierung als Chance und Herausforderung (Quelle: Eigene Darstellung $\left(\mathrm{n}_{\mathrm{DE}}=190, \mathrm{n}_{\mathrm{US}}=\right.$ 190))

\subsection{Fazit}

Sowohl in der wissenschaftlichen Literatur als auch in der Praxisliteratur werden für verschiedenste Branchen die Auswirkungen der Digitalisierung intensiv diskutiert. Greifbar gemacht werden die Auswirkungen durch Markt- und Wettbewerbsanalysen sowie eine Vielzahl von Erfahrungsberichten und Fallstudien zu Unternehmen, die das Ziel verfolgen, die Veränderungen ihrer Branche durch digitale Transformationsinitiativen zu gestalten oder sich diesen anzupassen. Die Ergebnisse der Umfrage unter 380 deutschen und US-amerikanischen Unternehmen zeigt, dass die Digitalisierung von der überwiegenden Zahl der Unternehmen in der Stichprobe als eine große Chance wahrgenommen wird. Wo Licht ist, ist jedoch auch Schatten, denn mit der Digitalisierung werden ebenso große Herausforderungen verbunden. Wie der Vergleich von Abbildung 2 und Abbildung 3 zeigt, dass die deutschen und US-amerikanischen Teilnehmer der Umfrage die mit der Digitalisierung verbundenen Herausforderungen sehr unterschiedlich einschätzen. Ein sehr großer Anteil der deutschen Unternehmen sieht in der konkreten Umsetzung von Digitalisierungsinitiativen eine besondere Herausforderung. Der Anteil der Unternehmen, der die Gefahr der Verdrängung von Unternehmen oder den Markteintritt neuer Wettbewerber als hoch einschätzt, fällt hingegen erheblich geringer aus. Umgekehrt sieht 
es bei den US-amerikanischen Unternehmen in der Stichprobe aus. Im Vergleich zu den deutschen Unternehmen schätzen erheblich weniger US-amerikanische Unternehmen die Umsetzung von Digitalisierungsinitiativen als große Herausforderung ein. Die weit größere Herausforderung scheinen die US-amerikanischen Unternehmen in der Gefahr der Verdrängung oder in neuen Wettbewerbern zu sehen.

Mögliche Erklärungen könnten in den unterschiedlichen Regularien beider Länder, Unterschieden in der Dynamik und Größe der Gründerszenen und der größeren gesamtwirtschaftlichen Rolle liegen, die der Dienstleistungssektor in den USA spielt. So werden die Markteintrittsbarrieren in einigen Branchen durch die deutsche Gesetzgebung signifikant erhöht. Ein Beispiel ist das Personenbeförderungsgesetz, das unter anderem eine Ortskenntnisprüfung voraussetzt, bevor ein Fahrer Personen gewerblich befördern darf. Damit kann der Fahrdienstvermittler Uber sein aus den USA bekanntes Geschäftsmodell nicht in Deutschland umsetzen ${ }^{8}$. Darüber hinaus befindet sich mit dem Silicon Valley eines der weltweit größten Innovationszentren der Welt in den USA. In Deutschland fällt es Startups schwerer an Kapital zu gelangen, um neue Geschäftsideen zu finanzieren und ein schnelles Wachstum zu ermöglichen (Roland Berger, 2016, S. 15). Aufgrund der hohen Dynamik in der US-amerikanischen Gründerszene sehen sich etablierte US-amerikanische Unternehmen vermutlich schneller mit potentiellen neuen Wettbewerbern konfrontiert, als etablierte deutsche Unternehmen. Nicht zuletzt ist davon auszugehen, dass die Rolle, die der Dienstleistungssektor in den beiden Ländern spielt, einen Einfluss auf die wahrgenommene Bedrohungslage hat. Der Dienstleistungssektor leistet in den USA einen erheblich höheren Beitrag zum BIP als in Deutschland ${ }^{9,10}$. Laut der Ergebnisse einer Expertenbefragung sind die größten Veränderungen durch die Digitalisierung gerade in diesem Sektor zu erwarten (Grossman, 2016).

\subsection{Literaturverzeichnis}

Barrett, C., \& Bradshaw, T. (2011). Burberry in Step with Digital Age. Financial Times. Burberry. (2017). Burberry Annual Report 2016/2017, London, England.

Burgelman, R. A., Siegel, R. E., \& Luther, J. (2014). Axel Springer in 2014: Strategic Leadership of the Digital Media Transformation. URL:

\footnotetext{
${ }^{8}$ www.spiegel.de/wirtschaft/unternehmen/fahrdienst-uber-uberpop-bleibt-in-deutschland-verboten-a1096768.html, zugegriffen am 11.08.2017

${ }^{9}$ de.statista.com/statistik/daten/studie/36846/umfrage/anteil-der-wirtschaftsbereiche-am-bruttoinlandsprodukt/, zugegriffen am 11.08.2017

${ }^{10} \mathrm{de}$.statista.com/statistik/daten/studie/165838/umfrage/anteile-der-wirtschaftssektoren-am-bruttoinlandsprodukt-der-usa/, zugegriffen am 11.08.2017
} 
https://www.gsb.stanford.edu/faculty-research/case-studies/axel-springer-2014-

strategic-leadership-digital-media-transformation, Stanford, CA, USA.

BVDM. (2015). Jahresbericht 2014/2015. URL: http://www.bvdm-

online.de/fileadmin/Jahresberichte/Jahresbericht 2014 2015.pdf, Berlin: B. D. u. M.

e.V.

Capgemini Consulting. (2012a). The Digital Advantage: How Digital Leaders Outperform

Their Peers in Every Industry: T. M. C. f. D. B. Capgemini Consulting.

Capgemini Consulting. (2012b). Digital Leadership - An Interview with Angela Ahrendts

CEO of Burberry. URL: https://www.capgemini.com/consulting/resources/digitalleadership-an-interview-with-angela-ahrendts/

Dishman, L. (2011). Burberry's "Tweetwalk" Delivers to the Masses and Challenges Fashion's Old Guard. Forbes.

Europäische Kommission. (2015). Antitrust: Commission sends Statement of Objections to

Google on comparison shopping service. URL: http://europa.eu/rapid/pressrelease MEMO-15-4781 en.htm

Grossman, R. (2016). The Industries That Are Being Disrupted the Most by Digital. Harvard Business Review.

Hagan, L. (2015). Burberry Case Study: Digital Transformation. URL:

https://www.kingcontent.com.au/burberry-digital-case-study/

Kim, E. (2014, 12.10.2015). Mark Zuckerberg Wants To Build The 'Perfect Personalized

Newspaper' For Every Person In The World. Business Insider UK.

manager magazin. (2017). Rekord-Kartellstrafe gegen Google wegen Shopping-Suche.

manager magazin.

Müller, S., Böhm, M., Schröer, M., Bakhirev, A., Baiasu, B., Krcmar, H., \& Welpe, I. (2016).

Geschäftsmodelle in der digitalen Wirtschaft, Studien zum deutschen

Innovationssystem, Berlin, Deutschland: EFI.

OECD. (2015). Data-Driven Innovation: Big Data for Growth and Well-Being. Paris, Frankreich: OECD Publishing.

Roland Berger. (2016). Towards a Franco-German Digital Valley, München, Deutschland.

Statista. (2015). Absatz von Zeitungen in Deutschland in den Jahren 2003 bis 2013. URL:

http://de.statista.com.eaccess.ub.tum.de/statistik/daten/studie/3877/umfrage/anzahltaeglich-verkaufter-zeitungen-seit-2003/.

Zhu, F., \& Iansiti, M. (2012). Entry into Platform-Based Markets. Strategic Management Journal, 33(1), 88-106. doi:10.1002/smj.941 
Open Access Dieses Kapitel wird unter der Creative Commons Namensnennung 4.0 International Lizenz (http://creativecommons.org/licenses/by/4.0/deed.de) veröffentlicht, welche die Nutzung, Vervielfältigung, Bearbeitung, Verbreitung und Wiedergabe in jeglichem Medium und Format erlaubt, sofern Sie den/die ursprünglichen Autor(en) und die Quelle ordnungsgemäß nennen, einen Link zur Creative Commons Lizenz beifügen und angeben, ob Änderungen vorgenommen wurden.

Die in diesem Kapitel enthaltenen Bilder und sonstiges Drittmaterial unterliegen ebenfalls der genannten Creative Commons Lizenz, sofern sich aus der Abbildungslegende nichts anderes ergibt. Sofern das betreffende Material nicht unter der genannten Creative Commons Lizenz steht und die betreffende Handlung nicht nach gesetzlichen Vorschriften erlaubt ist, ist für die oben aufgeführten Weiterverwendungen des Materials die Einwilligung des jeweiligen Rechteinhabers einzuholen. 